\section{RISE-Based Adaptive Control of a Control Affine Uncertain Nonlinear System With Unknown State Delays}

\author{
Nitin Sharma, Shubhendu Bhasin, Qiang Wang, and \\ Warren E. Dixon
}

\begin{abstract}
A continuous robust adaptive control method is designed for a class of uncertain nonlinear systems with unknown constant time-delays in the states. Specifically, a robust adaptive control method and a delay-free gradient-based desired compensation adaptation law (DCAL) are utilized to compensate for unknown time-delays, linearly parameterizable uncertainties, and additive bounded disturbances for a general nonlinear system. Despite these disturbances, a Lyapunov Krasovskii-based analysis is used to conclude that the system output asymptotically tracks a desired time varying bounded trajectory.
\end{abstract}

Index Terms-Desired compensation adaptation law (DCAL).

\section{INTRODUCTION}

TIME-DELAYED systems are endemic to engineering systems, leading to degraded control performance and potential instability. Various controllers have been developed to address time-delay induced performance and stability issues as described in the survey papers [1]-[3] (and the hundreds of references therein) and relatively recent monographs such as [4]-[9]. Control synthesis and stability analysis methods for nonlinear time-delayed systems are often based on Lyapunov-Krasovskii (LK) functionals (cf. [10]-[13]). For example, in [11], an iterative procedure utilizing LK functionals for robust stabilization of a class of nonlinear systems with a triangular structure is developed. However, as stated in [14], the controller cannot be constructed from the given iterative procedure. Semi-global uniformly ultimately bounded (SUUB) results have been developed for time-delayed nonlinear systems [12], [13] by utilizing neural network-based control, where appropriate LK functionals are utilized to remove time delayed states. A discontinuous adaptive controller was recently developed in [10] for a nonlinear system with an unknown time delay to achieve a UUB result with the aid of LK functionals. However, the controllers designed in [10], [12] can become singular when the controlled state reaches zero, and an ad hoc control strategy is proposed to overcome the problem. Moreover, as stated in [15] and [16], the control design procedure described in [13] cannot be generalized for nth order nonlinear systems. Robust output feedback based controllers have also been considered for linear systems [17]

Manuscript received July 22, 2010; revised February 22, 2011; accepted July 07, 2011. Date of publication August 30, 2011; date of current version December 29, 2011. Recommended by Associate Editor X. Chen.

N. Sharma is with the Department of Physiology, University of Alberta, Edmonton, AB, T6G 2E1, Canada (e-mail: nitin2@ualberta.ca).

S. Bhasin is with the Department of Mechanical and Aerospace Engineering, University of Florida, Gainesville FL 32611-6250 USA (e-mail: sbhasin@ufl. edu).

Q. Wang is with the Department of Electrical and Computer Engineering, University of Florida, Gainesville FL 32611-6250 USA (e-mail: qiangw @ufl. edu).

W. E. Dixon is with the Department of Mechanical and Aerospace Engineering and the Department of Electrical and Computer Engineering, University of Florida, Gainesville FL 32611-6250 USA (e-mail: wdixong@ufl.edu).

Color versions of one or more of the figures in this technical note are available online at http://ieeexplore.ieee.org.

Digital Object Identifier 10.1109/TAC.2011.2166314 and nonlinear systems [18] with delay, yielding UUB results in the presence of disturbances.

Sliding mode control (SMC) has also been utilized for time delayed systems in [19]-[23]. SMC-based control was also developed in [24]-[26] for switched linear systems with delay. However, utilizing SMC still poses a challenging design and computation problem when delays are present in the states [2], [3]. Moreover, the discontinuous sign function present in SMC often gives rise to undesirable chattering during practical applications. Motivated to address the discontinuities in SMC, a continuous adaptive sliding mode strategy is designed in [27] for nonlinear plants with unknown state delays, where an LK functional along with a discontinuous Lyapunov function is proposed for the stability analysis.

Robust control methods are prevalent in the aforementioned literature (with noted exceptions in [12], [13]). The development of adaptive control methods has been stymied (in part) due to the challenge of developing a delay-free adaptive update law. This technical note considers a dynamic system that is assumed to contain general unstructured uncertainties (where robust control methods are applied) and structured (linear in the uncertain parameters) uncertainty. For this general class of systems, potential time-delays in the regression matrix of an adaptive update law are avoided through the use of a desired compensation adaptive law (DCAL) technique and strategic segregation of terms in the open-loop error system, which facilitate the control design and stability analysis. A continuous Robust Integral of the Sign of the Error (RISE) structure [28], [29] is also included to compensate for the unstructured uncertainties. Instead of a typical UUB result, the continuous RISE method along with the novel adaptive update law and the appropriate choice of an LK functional are used to yield asymptotic tracking. Simulation results are provided to illustrate the performance of developed method for the typical regenerative chatter problem in high speed metal cutting.

\section{PRoblem Formulation}

Consider a class of uncertain nonlinear systems with an unknown state delay as [10]

$$
\begin{aligned}
\dot{x}_{1}= & x_{2} \\
\dot{x}_{n-1}(t)= & x_{n} \\
\dot{x}_{n}(t)= & f(x(t))+\delta_{1}(x(t))+g(x(t-\tau)) \\
& +\delta_{2}(x(t-\tau))+d(t)+b u(t) \\
y= & x_{1} .
\end{aligned}
$$

In (1), $f(x(t)), \delta_{1}(x(t)) \in \mathbb{R}^{m}$ are unknown functions, $g(x(t-\tau))$, $\delta_{2}(x(t-\tau)) \in \mathbb{R}^{m}$ are unknown time-delayed functions, $\tau \in \mathbb{R}^{+}$is an unknown constant arbitrarily large time delay, $d(t) \in \mathbb{R}^{m}$ is a bounded disturbance, $b \in \mathbb{R}^{+}$is an unknown constant, $u(t) \in \mathbb{R}^{m}$ is the control input, and $x(t) \triangleq$ $\left[\begin{array}{lll}x_{1}^{T} & x_{2}^{T} \ldots & x_{n}^{T}\end{array}\right]^{T} \in \mathbb{R}^{m n}$ denote system states, where $x(t)$ is assumed to be measurable. Also the following assumptions and notations will be exploited in the subsequent development.

Notation: Throughout the technical note, a time dependent delayed function is denoted as $x(t-\tau)$ or $x_{\tau}$, and a time dependent function (without time delay) is denoted as $x(t)$ or $x$. Assumption 1: The unknown functions $b^{-1} f(x), b^{-1} g\left(x_{\tau}\right)$ are linearly parameterizable, i.e., $b^{-1} f(x)=Y_{1}(x) \theta_{1}, b^{-1} g\left(x_{\tau}\right)=Y_{2}\left(x_{\tau}\right) \theta_{2}$, where $Y_{1}(\cdot) \in$ $\mathbb{R}^{m \times p_{1}}, Y_{2}(\cdot) \in \mathbb{R}^{m \times p_{2}}, \theta_{1} \in \mathbb{R}^{p_{1} \times 1}, \theta_{2} \in \mathbb{R}^{p_{2} \times 1}$ are constant unknown parameter vectors, and $p_{1}, p_{2}$ are positive integers. The regression matrix $Y_{2}\left(x_{\tau}\right)$ can not be computed due to the unknown time delay present in the state. Assumption 2: If $x(t) \in \mathcal{L}_{\infty}$, then $g(x), \delta_{1}(x)$, 
$\delta_{2}(x)$ are bounded. Moreover, the first and second partial derivatives of $g(x), \delta_{1}(x), \delta_{2}(x)$ with respect to $x(t)$ exist and are bounded (see [10], [12], [27]). Assumptions 1 and 2 are properties of many practical systems, such as typical Euler-Lagrange dynamics [30], [31] Assumption 3: The disturbance term $d(t)$ and its first two time derivatives are bounded. Assumption 4: The desired trajectory is designed such that $y_{d}(t), y_{d}^{i}(t) \in \mathcal{L}_{\infty}$, where $y_{d}^{(i)}(t)$ denotes the $i$ th time derivative for $i=1,2, \ldots, n+2$.

\section{ERROR SYSTEM DEVELOPMENT}

The control objective is to ensure that the output $y(t) \in \mathbb{R}^{m}$ tracks a desired time-varying trajectory $y_{d}(t) \in \mathbb{R}^{m}$ despite uncertainties in the system and an unknown time delay in the state. To quantify the objective, a tracking error, denoted by $e_{1}(t) \in \mathbb{R}^{m}$ and filtered tracking errors $e_{2}(t), \ldots, e_{n}(t), r(t) \in \mathbb{R}^{m}$ are defined as

$$
\begin{aligned}
e_{1}(t) & \triangleq y(t)-y_{d}(t) \\
e_{2}(t) & \triangleq \dot{e}_{1}(t)+\alpha_{1} e_{1}(t) \\
e_{i}(t) & \triangleq \dot{e}_{i-1}(t)+\alpha_{i-1} e_{i-1}(t)+e_{i-2} \\
r(t) & \triangleq \dot{e}_{n}(t)+\alpha_{n} e_{n}(t)
\end{aligned}
$$

where $\alpha_{1}, \ldots, \alpha_{n} \in \mathbb{R}^{+}$denote constant control gains. As defined in (5), the filtered tracking error $r(t)$ is not measurable since the expression depends on $\dot{x}_{n}(t)$. However, $e_{1}(t), \ldots, e_{n}(t) \in \mathbb{R}^{m}$ are measurable because (4) can be expressed in terms of the tracking error $e_{1}(t)$ as

$$
e_{i}(t)=\sum_{j=0}^{i-1} a_{i j} e_{1}^{(j)}, \quad i=2, \ldots, n
$$

where $a_{i j} \in \mathbb{R}^{+}$are constants obtained from substituting (6) in (4) and comparing coefficients, and $a_{i j}=1, j=i-1$ [32]. Using (2)-(6), the open loop error system can be written as

$$
r=y^{(n)}-y_{d}^{(n)}+l
$$

where $l\left(e_{1}, \dot{e}_{1}, \ldots, e_{1}^{(n-1)}\right) \in \mathbb{R}^{m}$ is a function of known and measurable terms, defined as

$$
l=\sum_{j=0}^{n-2} a_{n j}\left(e_{1}^{(j+1)}+\alpha_{n} e_{1}^{(j)}\right)+\alpha_{n} e_{1}^{(n-1)} .
$$

The open-loop tracking error system can be developed by premultiplying (7) by $b^{-1}$ and using (1) and Assumption 1 as

$$
\begin{aligned}
b^{-1} r=Y_{1}(x) \theta_{1}+b^{-1} \delta_{1}(x)+ & Y_{2}\left(x_{\tau}\right) \theta_{2}+b^{-1} \delta_{2}\left(x_{\tau}\right) \\
& +b^{-1} d+u-b^{-1} y_{d}^{(n)}+b^{-1} l .
\end{aligned}
$$

In the subsequent development, a DCAL-based update law is developed in terms of $Y_{2}(\cdot)$ without a state delay. After some algebraic manipulation, the expression in (8) can be rewritten as

$$
\begin{aligned}
b^{-1} r=b^{-1} l+S_{1}+S_{2}+W+b^{-1} d+Y_{1}\left(x_{d}\right) \theta_{1} \\
+Y_{2}\left(x_{d}\right) \theta_{2}+u
\end{aligned}
$$

where the auxiliary functions $S_{1}\left(x_{d}, x\right), \quad S_{2}\left(x_{d \tau}, x_{\tau}\right)$, $W\left(x_{d}, x_{d \tau}, y_{d}^{(n)}\right) \in \mathbb{R}^{m}$ are defined as

$$
\begin{aligned}
S_{1}= & Y_{1}(x) \theta_{1}-Y_{1}\left(x_{d}\right) \theta_{1}+b^{-1} \delta_{1}(x) \\
& -b^{-1} \delta_{1}\left(x_{d}\right)
\end{aligned}
$$

$$
\begin{aligned}
S_{2}= & Y_{2}\left(x_{\tau}\right) \theta_{2}-Y_{2}\left(x_{d \tau}\right) \theta_{2}+b^{-1} \delta_{2}\left(x_{\tau}\right) \\
& -b^{-1} \delta_{2}\left(x_{d \tau}\right) \\
W= & b^{-1} \delta_{1}\left(x_{d}\right)+b^{-1} \delta_{2}\left(x_{d \tau}\right)-Y_{2}\left(x_{d}\right) \theta_{2} \\
& +Y_{2}\left(x_{d \tau}\right) \theta_{2}-b^{-1} y_{d}^{(n)}
\end{aligned}
$$

where $x_{d} \triangleq\left[y_{d}^{T} \dot{y}_{d}^{T} \ldots\left(y_{d}^{(n-1) T}\right)\right]^{T} \in \mathbb{R}^{m n}$ denotes a column vector containing the desired trajectory and its derivatives. The grouping of terms and structure of (9) is motivated by the subsequent stability analysis and the need to develop an adaptive update law that is invariant to the unknown time delay. The auxiliary function $S_{1}\left(x_{d}, x\right)$ is defined such that it contains terms that are not time-delay dependent. The auxiliary function $S_{2}\left(x_{d \tau}, x_{\tau}\right)$ is introduced to isolate the time-delayed states, and $W\left(x_{d}, x_{d \tau}, \dot{x}_{d}\right)$ is isolated because it only contains functions of the desired trajectory.

Based on the open-loop error system in (9), the control input $u(t) \in$ $\mathbb{R}^{m}$ is designed as

$$
u=-Y_{1}\left(x_{d}\right) \hat{\theta}_{1}-Y_{2}\left(x_{d}\right) \hat{\theta}_{2}-\mu .
$$

In (13), $\mu(t) \in \mathbb{R}^{m}$ is the generalized solution to

$$
\dot{\mu}=\left(k_{s}+1\right) r+\beta \operatorname{sgn}\left(e_{n}\right), \quad \mu(0)=0
$$

where $k_{s}, \beta \in \mathbb{R}^{+}$are known constant gains. In (13), $\hat{\theta}_{1}(t) \in \mathbb{R}^{p_{1}}$, $\hat{\theta}_{2}(t) \in \mathbb{R}^{p_{2}}$ denote parameter estimate vectors generated as

$$
\dot{\hat{\theta}}_{1}=\Gamma_{1} \dot{Y}_{1}^{T}\left(x_{d}\right) r \quad \dot{\hat{\theta}}_{2}=\Gamma_{2} \dot{Y}_{2}^{T}\left(x_{d}\right) r
$$

where $\Gamma_{1} \in \mathbb{R}^{p_{1} \times p_{1}}, \Gamma_{2} \in \mathbb{R}^{p_{2} \times p_{2}}$ are known, constant, diagonal, positive definite adaptation gain matrices. In (15), $\dot{Y}_{2}^{T}\left(x_{d}\right)$ does not depend on the time delayed desired state. This delay free law is achieved by isolating the delayed term $Y_{2}\left(x_{d \tau}\right) \theta_{2}$ in the auxiliary signal $W\left(x_{d}, x_{d \tau}, y_{d}^{(n)}\right)$ in (12). The adaptation laws in (15) depend on the unmeasurable signal $r(t)$, but by using the fact that $\dot{Y}_{1}\left(x_{d}\right)$, $\dot{Y}_{2}\left(x_{d}\right)$ are functions of the known desired trajectory, integration by parts can be used to implement $\hat{\theta}_{i}(t)$ for $i=1,2$ where only $e_{n}(t)$ is required as

$$
\begin{aligned}
\hat{\theta}_{i}=\hat{\theta}_{i}(0)+ & \left.\Gamma_{i} \dot{Y}_{i}^{T}\left(x_{d}\right) e_{n}(\sigma)\right|_{0} ^{t} \\
& -\Gamma_{i} \int_{0}^{t}\left\{\ddot{Y}_{i}^{T}\left(x_{d}\right) e_{n}(\sigma)-\alpha_{n} \dot{Y}_{i}^{T}\left(x_{d}\right) e_{n}(\sigma)\right\} d \sigma .
\end{aligned}
$$

The closed-loop error system can be developed by substituting (13) into (9) as

$$
\begin{array}{r}
b^{-1} r=b^{-1} l+S_{1}+S_{2}+W+b^{-1} d-\mu+Y_{1}\left(x_{d}\right) \tilde{\theta}_{1} \\
+Y_{2}\left(x_{d}\right) \tilde{\theta}_{2}
\end{array}
$$

where $\tilde{\theta}_{i}$ for $i=1,2$ are the parameter estimation error vectors defined as $\tilde{\theta}_{i}=\theta_{i}-\hat{\theta}_{i}$. To facilitate the subsequent stability analysis and to more clearly illustrate how the Robust Integral of the Sign of the Error (RISE) structure in (14) is used to reject the disturbance terms, the time derivative of (17) is determined as

$$
\begin{aligned}
b^{-1} \dot{r}=\tilde{N}+N_{d}-e_{n}+\dot{Y}_{1}\left(x_{d}\right) \tilde{\theta}_{1} & +\dot{Y}_{2}\left(x_{d}\right) \tilde{\theta}_{2} \\
& -\left(k_{s}+1\right) r-\beta \operatorname{sgn}\left(e_{n}\right)
\end{aligned}
$$

where the auxiliary functions $\tilde{N}\left(e_{1}, \ldots e_{n}, r, e_{1 \tau}, \ldots, e_{n \tau}, r_{\tau}\right)$, $N_{d}\left(\dot{x}_{d}, \ddot{x}_{d}, t\right) \in \mathbb{R}^{m}$ are defined as

$$
\begin{aligned}
\tilde{N}= & b^{-1} \dot{l}+\dot{S}_{1}+\dot{S}_{2}+e_{n}-Y_{1}\left(x_{d}\right) \Gamma_{1} \dot{Y}_{1}\left(x_{d}\right) r \\
& -Y_{2}\left(x_{d}\right) \Gamma_{2} \dot{Y}_{2}\left(x_{d}\right) r \\
N_{d}= & \dot{W}+b^{-1} \dot{d} .
\end{aligned}
$$


Using Assumptions 2, 3, and 4, $N_{d}\left(x_{d}, \dot{x}_{d}, \ddot{x}_{d}, t\right)$ and its time derivative can be upper bounded as

$$
\left\|N_{d}\right\| \leq \zeta_{N_{d}}, \quad\left\|\dot{N}_{d}\right\| \leq \zeta_{\dot{N}_{d}}
$$

where $\zeta_{N_{d}}, \quad \zeta_{\dot{N}_{d}} \in \mathbb{R}^{+}$are known constants, and $\tilde{N}\left(e_{1}, \ldots e_{n}, r, e_{1 \tau}, \ldots, e_{n \tau}, r_{\tau}\right)$ can be upper bounded as

$$
\|\tilde{N}\| \leq \rho_{1}(\|z\|)\|z\|+\rho_{2}\left(\left\|z_{\tau}\right\|\right)\left\|z_{\tau}\right\|
$$

where $z(t) \in \mathbb{R}^{(n+1) m}$ is defined as

$$
z=\left[\begin{array}{lllllll}
e_{1}^{T} & e_{2}^{T} & \cdot & \cdot & e_{n}^{T} & r^{T}
\end{array}\right]^{T}
$$

and the known bounding functions $\rho_{1}(\|z\|), \rho_{2}\left(\left\|z_{\tau}\right\|\right) \in \mathbb{R}$ are positive, globally invertible, and nondecreasing functions. Note that the upper bound for the auxiliary function $\tilde{N}\left(e_{1}, e_{2}, e_{1 \tau}, e_{2 \tau}\right)$ in (21) is segregated into delay free and delayed upper bound functions. Motivation for this segregation of terms is to eliminate the delay dependent term through the use of an LK functional in the stability analysis. Specifically, let $Q(t) \in \mathbb{R}$ denote an LK functional defined as

$$
Q=\frac{1}{2 k_{s}} \int_{t-\tau}^{t} \rho_{2}^{2}(\|z(\sigma)\|)\|z(\sigma)\|^{2} d \sigma
$$

where $k_{s}$ and $\rho_{2}(\cdot)$ are introduced in (14) and (21), respectively.

\section{Stability ANALYSIS}

Theorem 1: The controller given in (13)-(15) ensures that all system signals are bounded under closed-loop operation. The tracking error is regulated in the sense that $\left\|e_{1}(t)\right\| \rightarrow 0$ as $t \rightarrow \infty$, provided the control gain $k_{s}$ introduced in (14) is selected sufficiently large, and $\alpha_{n-1}, \alpha_{n}$, and $\beta$ are selected according to the following sufficient conditions:

$$
\beta>\left(\zeta_{N_{d}}+\frac{1}{\alpha} \zeta_{\dot{N}_{d}}\right), \quad \alpha_{n-1}, \alpha_{n}>\frac{1}{2}
$$

where $\alpha_{n-1}, \alpha_{n}$ are introduced in (4) and (5), respectively; $\beta$ is introduced in (14); and $\zeta_{N_{d}}$ and $\zeta_{\dot{N}_{d}}$ are introduced in (20).

Proof: Let $\mathcal{D} \subset \mathbb{R}^{(n+1) m+p_{1}+p_{2}+2}$ be a domain containing $y(t)=0$, where $y(t) \in \mathbb{R}^{(n+1) m+2+p_{1}+p_{2}}$ is defined as

$$
y(t) \triangleq\left[\begin{array}{lllll}
z^{T} & \sqrt{P(t)} & \sqrt{Q(t)} & \tilde{\theta}_{1}^{T} & \tilde{\theta}_{2}^{T}
\end{array}\right]^{T}
$$

where $\tilde{\theta}_{1}(t), \tilde{\theta}_{2}(t)$ are introduced in (17), $z(t)$ and $Q(t)$ are defined in (22) and (23), respectively, and the auxiliary function $P(t) \in \mathbb{R}$ is the generalized solution to the differential equation

$$
\begin{aligned}
& \dot{P}(t)=-r^{T}\left(N_{d}(t)-\beta \operatorname{sgn}\left(e_{n}\right)\right), \\
& P(0)=\beta \sum_{i=1}^{m}\left|e_{n i}(0)\right|-e_{n}(0)^{T} N_{d}(0) .
\end{aligned}
$$

Provided the sufficient conditions stated in Theorem 1 are satisfied, then $P(t) \geq 0$ [29].

Let $V_{L}(y, t): \mathcal{D} \times[0, \infty) \rightarrow \mathbb{R}$ denote a Lipschitz continuous regular positive definite functional defined as

$$
\begin{aligned}
V(y, t) \triangleq \frac{1}{2} e_{1}^{T} e_{1}+\frac{1}{2} e_{2}^{T} e_{2}+ & \cdots+\frac{1}{2} e_{n}^{T} e_{n}+\frac{1}{2} r^{T} b^{-1} r \\
& +P+Q+\frac{1}{2} \tilde{\theta}_{1}^{T} \Gamma_{1}^{-1} \tilde{\theta}_{1}+\frac{1}{2} \tilde{\theta}_{2}^{T} \Gamma_{2}^{-1} \tilde{\theta}_{2}
\end{aligned}
$$

which satisfies the following inequalities

$$
U_{1}(y) \leq V(y, t) \leq U_{2}(y)
$$

provided the sufficient conditions introduced in Theorem 1 are satisfied. In (27), $U_{1}(y), U_{2}(y) \in \mathbb{R}$ are continuous, positive definite functions defined as $U_{1}(y)=\gamma_{1}\|y\|^{2} U_{2}(y)=\gamma_{2}\|y\|^{2}$, where $\gamma_{1}$, $\gamma_{2} \in \mathbb{R}^{+}$are known constants. After taking the time derivative of (28), $\dot{V}_{L}(y, t)$ can be expressed as

$$
\begin{aligned}
\dot{V}_{L}(y, t) \triangleq e_{1}^{T} \dot{e}_{1}+e_{2}^{T} \dot{e}_{2} & +\cdots+e_{n}^{T} \dot{e}_{n}+r^{T} b^{-1} \dot{r} \\
& +\dot{P}+\dot{Q}+\tilde{\theta}_{1}^{T} \Gamma_{1}^{-1} \dot{\theta}_{1}+\tilde{\theta}_{2}^{T} \Gamma_{2}^{-1} \dot{\theta}_{2} .
\end{aligned}
$$

Some of the differential equations describing the closed-loop system for which the stability analysis is being performed have discontinuous right-hand sides denoted by $f(y, t) \in \mathbb{R}^{(n+1) m+p_{1}+p_{2}+2}$ in the set $\left\{(y, t) \mid e_{n}=0\right\}$. As described in [33], the existence of Filippov's generalized solution can be established for $\dot{y}=f(y, t)$. From [33], an absolute continuous Filippov solution $y(t)$ exists almost everywhere (a.e.) so that $\dot{y} \in K[f](y, t)$ a.e. where $K[\cdot]$ is defined as [34] $K[f](y) \triangleq \bigcap_{\delta>0} \bigcap_{\mu=0} \overline{c o} f(B(x, \delta)-N)$, where $\bigcap_{\mu N=0}$ denotes the intersection of all sets $N$ of Lebesgue measure zero, $\overline{c o}$ denotes convex closure, and $B(x, \delta)$ represents a ball of radius $\delta$ around $x$. Under Filippov's framework, a generalized Lyapunov stability theory can be used (see [34], [35] for further details) to establish strong stability of the closed-loop system. The generalized time derivative of (28) exists a.e., and $\dot{V}_{L}(y, t) \in^{a . e \cdot} \dot{\tilde{V}}_{L}(y, t)$ where

$$
\begin{aligned}
& \dot{\tilde{V}}_{L}(y, t)=\bigcap_{\xi \in \partial V_{L}(y, t)} \xi^{T} K\left[\begin{array}{lll}
\dot{e}_{1} & \dot{e}_{2} & \cdot
\end{array}\right. \\
& \text { - } \dot{e}_{n} \quad \frac{1}{2} P^{-1 / 2} \dot{P} \quad \frac{1}{2} Q^{-1 / 2} \dot{Q} \\
& \left.\begin{array}{ll}
\tilde{\theta}_{1} & \dot{\tilde{\theta}}_{2}
\end{array}\right]^{T}
\end{aligned}
$$

where $\partial V$ is the generalized gradient of $V$ [35]. After utilizing (3), (4), (5), (19), (26), adaptation laws in (15) and the time derivative of $Q(t)$ in (23), the expression in (29) can be rewritten as

$$
\begin{aligned}
& \dot{\tilde{V}}_{L}(y, t) \subset-\alpha_{1} e_{1}^{T} e_{1}-\cdots-\alpha_{n} e_{n}^{T} e_{n}+e_{n}^{T} e_{n-1} \\
& \quad+e_{n}^{T} r+r^{T} \tilde{N}+r^{T} N_{d}-r^{T} e_{n}+r^{T} \dot{Y}_{1}\left(x_{d}\right) \tilde{\theta}_{1} \\
& \quad+r^{T} \dot{Y}_{2}\left(x_{d}\right) \tilde{\theta}_{2}-\left(k_{s}+1\right)\|r\|^{2} \\
& \quad-\beta r^{T} K\left[\operatorname{sgn}\left(e_{n}\right)\right]-r^{T} N_{d}(t)+\beta r^{T} K\left[\operatorname{sgn}\left(e_{n}\right)\right] \\
& \quad-\tilde{\theta}_{1}^{T} \dot{Y}_{1}^{T}\left(x_{d}\right) r-\tilde{\theta}_{2} \dot{Y}_{2}^{T}\left(x_{d}\right) r+ \\
& \quad \frac{1}{2 k_{s}}\left(\rho_{2}^{2}(\|z\|)\|z\|^{2}-\rho_{2}^{2}\left(\left\|z_{\tau}\right\|\right)\left\|z_{\tau}\right\|^{2}\right)
\end{aligned}
$$

where $K\left[\operatorname{sgn}\left(e_{n}\right)\right]=1$ if $e_{n}>0,[-1,1]$ if $e_{n}=0,-1$ if $e_{n}<0$. Cancelling common terms and using (21) yields

$$
\begin{aligned}
& \dot{\tilde{V}}_{L}(y, t) \leq-\sum_{i=1}^{n} \alpha_{i}\left\|e_{i}\right\|^{2}+e_{n-1}^{T} e_{n}-\|r\|^{2}-k_{s}\|r\|^{2} \\
& \quad+\rho_{2}\left(\left\|z_{\tau}\right\|\right)\left\|z_{\tau}\right\|\|r\|+\rho_{1}(\|z\|)\|z\|\|r\| \\
& +\frac{\rho_{2}^{2}(\|z\|)\|z\|^{2}}{2 k_{s}}-\frac{\rho_{2}^{2}\left(\left\|z_{\tau}\right\|\right)\left\|z_{\tau}\right\|^{2}}{2 k_{s}}
\end{aligned}
$$

After applying Young's inequality to determine that

$$
\begin{aligned}
\rho_{2}\left(\left\|z_{\tau}\right\|\right)\left\|z_{\tau}\right\|\|r\| & \leq \frac{\rho_{2}^{2}\left(\left\|z_{\tau}\right\|\right)\left\|z_{\tau}\right\|^{2}}{2 k_{s}}+\frac{k_{s}}{2}\|r\|^{2}, \\
e_{n-1}^{T} e_{n} & \leq \frac{1}{2}\left(\left\|e_{n-1}\right\|^{2}+\left\|e_{n}\right\|^{2}\right)
\end{aligned}
$$


the expression in (31) can be written as

$$
\begin{gathered}
\dot{\tilde{V}}_{L}(y, t) \leq-\sum_{i=1}^{n-2} \alpha_{i}\left\|e_{i}\right\|^{2}-\left(\alpha_{n-1}-\frac{1}{2}\right)\left\|e_{n-1}\right\|^{2} \\
-\left(\alpha_{n}-\frac{1}{2}\right)\left\|e_{n}\right\|^{2}-\|r\|^{2}-\frac{k_{s}}{2}\|r\|^{2} \\
+\rho_{1}(\|z\|)\|z\|\|r\|+\frac{\rho_{2}^{2}(\|z\|)\|z\|^{2}}{2 k_{s}}
\end{gathered}
$$

After completing the squares, the expression in (33) can be written as

$$
\dot{\tilde{V}}_{L}(y, t) \leq-\left[\gamma_{3}-\frac{\rho^{2}(\|z\|)}{2 k_{s}}\right]\|z\|^{2}
$$

where $\rho^{2}(\|z\|) \in \mathbb{R}$ is defined as $\rho^{2}(\|z(t)\|)=\rho_{1}^{2}(\|z(t)\|)+$ $\rho_{2}^{2}(\|z(t)\|)$, and $\gamma_{3} \triangleq \min \left[\alpha_{1}, \alpha_{2}, \ldots, \alpha_{n-2}, \alpha_{n-1}-1 / 2, \alpha_{n}-\right.$ $1 / 2,1]$. The bounding function $\rho(\|z\|)$ is a positive, globally invertible, and nondecreasing function that does not depend on the time-delay. The expression in (34) can be further upper bounded by a continuous, positive semi-definite function

$$
\dot{\tilde{V}}_{L}(y, t) \leq-U(y)=-c\|z\|^{2} \quad \forall y \subset \mathcal{D}
$$

for some positive constant $c$, where $\mathcal{D}$ $\left\{y(t) \in \mathbb{R}^{(n+1) m+p_{1}+p_{2}+2} \mid\|y\| \leq \rho^{-1}\left(\sqrt{2 \gamma_{3} k_{s}}\right)\right\}$.

Larger values of $k_{s}$ will expand the size of the domain $\mathcal{D}$. The inequalities in (27) and (35) can be used to show that $V(y, t) \in \mathcal{L}_{\infty}$ in $\mathcal{D}$; hence, $e_{1}, e_{2}, \ldots, e_{n}, \tilde{\theta}_{1}, \tilde{\theta}_{2} \in \mathcal{L}_{\infty}$ in $\mathcal{D}$. The closed-loop error systems can now be used to conclude all remaining signals are bounded in $\mathcal{D}$, and the definitions for $U(y)$ and $z(t)$ can be used to prove that $U(y)$ is uniformly continuous in $\mathcal{D}$. Let $\mathcal{S} \subset \mathcal{D}$ denote a set defined as

$$
\mathcal{S} \triangleq\left\{y(t) \in \mathcal{D} \mid U_{2}(y(t))<\gamma_{1}\left(\rho^{-1}\left(\sqrt{2 \gamma_{3} k_{s}}\right)\right)^{2}\right\} .
$$

The region of attraction in (36) can be made arbitrarily large to include any initial conditions by increasing the control gain $k_{s}$ (i.e., a semiglobal stability result), and hence

$$
c\|z\|^{2} \rightarrow 0 \quad \text { as } \quad t \rightarrow \infty \quad \forall y(0) \in \mathcal{S} .
$$

Based on the definition of $z(t)$, (37) can be used to show that $\left\|e_{1}(t)\right\| \rightarrow 0$ as $t \rightarrow \infty \forall y(0) \in \mathcal{S}$.

\section{Simulation}

To illustrate the performance of the designed RISE-based adaptive controller, we consider the following second order scalar nonlinear system that describes the chattering phenomenon during a metal cutting operation [5], [36]-[38]

$$
m \ddot{y}+c \dot{y}+k y=F(y, t)
$$

where $m, c, k$ are the effective mass, damping coefficient, and stiffness constant, respectively. The thrust force $F(y, t) \in \mathbb{R}$, defined as

$$
F(y, t)=k_{c} b(f-y+y(t-\tau))
$$

is a function of inner modulation $y(t) \in \mathbb{R}$ and outer modulation $y(t-$ $\tau) \in \mathbb{R}$, where $k_{c}, b, f$ are cutting stiffness, width of cut, and feed rate, respectively, and $\tau$ is the time delay due to the spindle rotation period. Under certain cutting conditions and in the absence of a control input the vibrations are unstable as depicted in Fig. 1. The following closed-

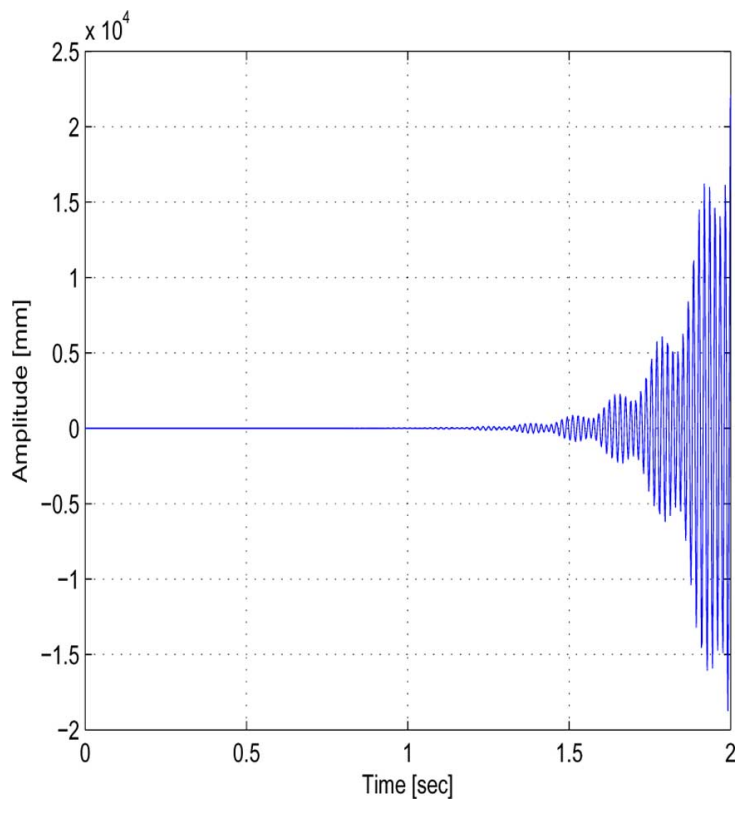

Fig. 1. Chatter instability in the absence of control.
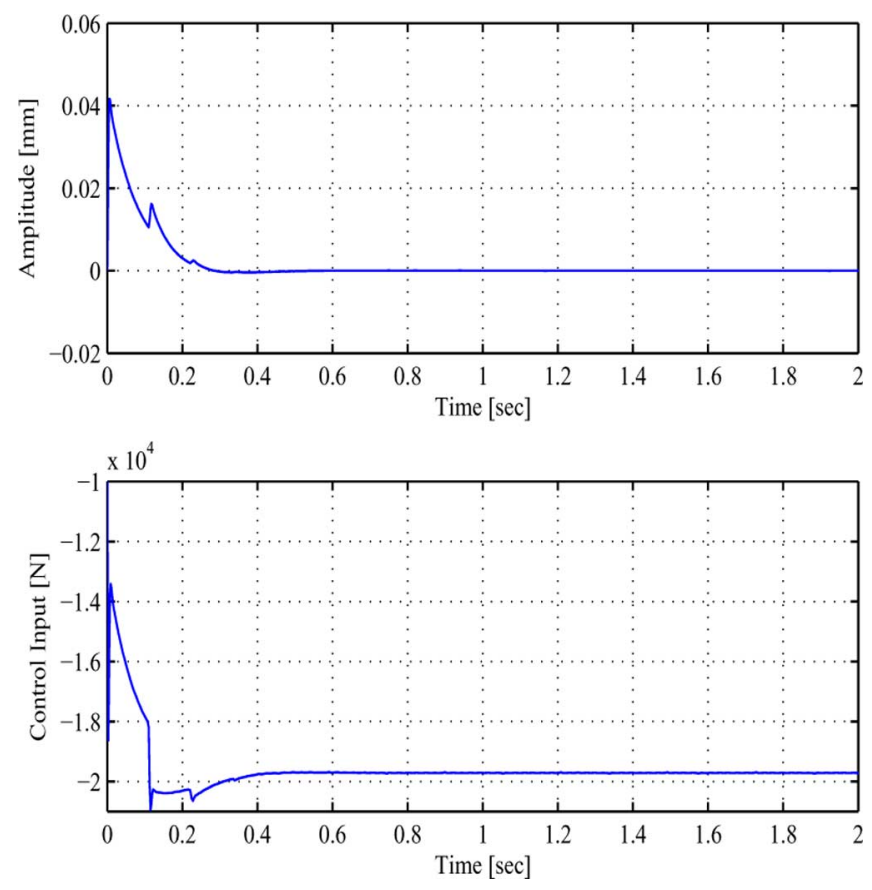

Fig. 2. Top plot shows the amplitude of vibrations and the bottom plot shows the control input.

loop system is simulated to depict the performance of the RISE-based adaptive controller:

$$
\begin{aligned}
\dot{x}_{1} & =x_{2}, \\
\dot{x}_{2} & =m^{-1}\left(-c \dot{y}-k y+k_{c} b(f-y+y(t-\tau))+u\right) \\
y & =x_{1}
\end{aligned}
$$

where $u(t) \in \mathbb{R}$ is the control input designed in (13). For the simulation, the unknown system parameters are chosen as: $m=1.16 \mathrm{~kg}$, 
$\tau=60 / \Omega$ sec $, \Omega=550 \mathrm{rpm}, k=m \omega^{2}, c=2 \mathrm{~m} \zeta \omega, \zeta=0.1$, $\omega=83 \pi, k_{c} / k=0.5, b=2 \mathrm{~mm}$, and $f=0.25 \mathrm{~mm}$ per revolution. Note that Assumptions 1 and 2 are satisfied for this practical system. From the results shown in Fig. 2, the controller is able to eliminate the tool workpiece chatter.

\section{CONCLUSION}

A continuous robust controller is utilized in conjunction with an adaptive controller for stabilizing a class of uncertain nonlinear systems with unknown constant state delays and bounded disturbances. By utilizing a DCAL-based method and segregating the necessary terms, the developed controller and the adaptive estimate law does not depend on the unknown time delay. Simulations are provided for the regenerative chatter metal cutting problem [1], to show the performance of the controller and applicability of assumptions. An LK functional is used to prove asymptotic tracking for the closed-loop nonlinear system. A remaining open problem is developing a continuous controller that can yield an asymptotic result despite time-varying state delays. Moreover, methods such as [17], [18] may also provide insight for the development of observer-based output feedback results that could potentially be extended to an adaptive controller.

\section{REFERENCES}

[1] R. Sipahi, S.-I. Niculescu, C. Abdallah, W. Michiels, and K. Gu, "Stability and stabilization of systems with time delay: Limitations and opportunities," IEEE Contr. Syst. Mag., vol. 31, no. 1, pp. 38-65, 2011.

[2] K. Gu and S. Niculescu, "Survey on recent results in the stability and control of time-delay systems," J. Dyn. Syst. Meas. Control, vol. 125, pp. 158-158, 2003.

[3] J.-P. Richard, "Time-delay systems: An overview of some recent advances and open problems," Automatica, vol. 39, no. 10, pp. 1667-1694, 2003.

[4] , J. Chiasson and J. Loiseau, Eds., Applications of Time Delay Systems. Berlin, Germany: Spring Verlag, 2007.

[5] K. Gu, V. L. Kharitonov, and J. Chen, Stability of Time-Delay Systems. Boston, MA: Birkhauser, 2003.

[6] M. Krstic, Delay Compensation for Nonlinear, Adaptive, and PDE Systems. New York: Springer, 2009.

[7] , J. Loiseau, W. Michiels, S. I. Niculescu, and R. Sipahi, Eds., Topics in Time Delay Systems: Analysis, Algorithms, and Control. Berlin, Germany: Springer Verlag, 2009.

[8] M. S. Mahmoud, Robust Control and Filtering for Time-Delay Systems. Orlando, FL: CRC Press, 2000.

[9] S.-I. Niculescu and K. Gu, Advances in Time-Delay Systems. Berlin, Germany: Springer Verlag, 2004.

[10] S. Ge, F. Hong, and T. Lee, "Robust adaptive control of nonlinear systems with unknown time delays," Automatica, vol. 41, no. 7, pp. 1181-1190, Jul. 2005

[11] S. Nguang, "Robust stabilization of a class of time-delay nonlinear systems," IEEE Trans. Autom. Control, vol. 45, no. 4, pp. 756-762, Apr. 2000.

[12] S. Ge, F. Hong, and T. H. Lee, "Adaptive neural network control of nonlinear systems with unknown time delays," IEEE Trans. Autom. Control, vol. 48, no. 11, pp. 2004-2010, Nov. 2003.

[13] D. Ho, L. Junmin, and Y. Niu, "Adaptive neural control for a class of nonlinearly parametric time-delay systems," IEEE Trans. Neural Netw., vol. 16, no. 3, pp. 625-635, May 2005.

[14] S. Zhou, G. Feng, and S. K. Nguang, "Comments on robust stabilization of a class time-delay nonlinear systems," IEEE Trans. Autom. Control, vol. 47, no. 9, pp. 1586-1586, Sep. 2002.

[15] S. J. Yoo, J. B. Park, and Y. H. Choi, "Comments on adaptive neural control for a class of nonlinearly parametric time-delay systems," IEEE Trans. Neural Networks, vol. 19, no. 8, pp. 1496-1498, Aug. 2008.

[16] D. Ho, J. Li, and Y. Niu, "Reply to comments on adaptive neural control for a class of nonlinearly parametric time-delay systems," IEEE Trans. Neural Networks, vol. 19, no. 8, pp. 1498-1498, Aug. 2008.

[17] A. Bobtsov and A. Nagovitsina, "Adaptive output control of linear time-varying systems," in Proc. IFAC Workshop Adaptation Learning Control Signal Processing, 2007, [CD ROM].
[18] A. Bobtsov, "Output stabilization of nonlinear systems under delay conditions," J. Comp. Syst. Sci. Int., vol. 47, no. 2, pp. 179-186, 2008.

[19] K. Shyu and J. Yan, "Robust stability of uncertain time-delay systems and its stabilization by variable structure control," Int. J. Control, vol. 57, no. 1, pp. 237-246, 1993.

[20] Y. Xia and Y. Jia, "Robust sliding-mode control for uncertain timedelay systems: An LMI approach," IEEE Trans. Autom. Control, vol. 48, no. 6, pp. 1086-1091, Jun. 2003.

[21] F. Gouaisbaut, W. Perruquetti, and J. P. Richard, "A sliding mode control for linear systems with input and state delays," in Proc. IEEE Conf. Decis. Control, Dec. 7-10, 1999, vol. 4, pp. 4234-4239.

[22] F. Gouaisbaut, M. Dambrine, and J. P. Richard, "Sliding mode control of TDS via functional surfaces," in Proc. IEEE Conf. Decision Control, Dec. 4-7, 2001, vol. 5, pp. 4630-4634.

[23] F. Gouaisbaut, M. Dambrine, and J. Richard, "Robust control of delay systems: A sliding mode control design via LMI," Syst. Control Lett., vol. 46, no. 4, pp. 219-230, 2002.

[24] J. Lian, J. Zhao, and G. Dimirovski, "Model reference adaptive integral sliding mode control for switched delay systems," Int. J. Innovative Comp., Inform. Control, vol. 4, pp. 2025-2032, 2008.

[25] J. Lian, J. Zhao, and G. M. Dimirovski, "Robust infinity sliding mode control for a class of uncertain switched delay systems," Int. J. Syst. Sci., vol. 40, no. 8, pp. 855-866, 2009.

[26] J. Lian, J. Zhao, and G. Dimirovski, "Integral sliding mode control for a class of uncertain switched nonlinear systems," Eur. J. Control, vol. 16 , no. 1, pp. 16-22, 2010.

[27] B. Mirkin, P.-O. Gutman, and Y. Shtessel, "Continuous model reference adaptive control with sliding mode for a class of nonlinear plants with unknown state delay," in Proc. Amer. Control Conf., 10-12, 2009, pp. $574-579$.

[28] P. M. Patre, W. MacKunis, C. Makkar, and W. E. Dixon, "Asymptotic tracking for systems with structured and unstructured uncertainties," IEEE Trans. Control Syst. Technol., vol. 16, no. 2, pp. 373-379, 2008.

[29] P. M. Patre, W. MacKunis, K. Kaiser, and W. E. Dixon, "Asymptotic tracking for uncertain dynamic systems via a multilayer neural network feedforward and RISE feedback control structure," IEEE Trans. Autom. Control, vol. 53, no. 9, pp. 2180-2185, Oct. 2008.

[30] W. E. Dixon, A. Behal, D. M. Dawson, and S. Nagarkatti, Nonlinear Control of Engineering Systems: A Lyapunov-Based Approach. Boston, MA: Birkhauser, 2003.

[31] R. Ortega, A. Lora, P. J. Nicklasson, and H. J. Sira-Ramirez, PassivityBased Control of Euler-Lagrange Systems: Mechanical, Electrical and Electromechanical Applications. New York: Springer, 1998.

[32] B. Xian, M. de Queiroz, and D. Dawson, "A continuous control mechanism for uncertain nonlinear systems," in Optimal Control, Stabilization and Nonsmooth Analysis, ser. Lecture Notes in Control and Information Sciences. Heidelberg, Germany: Springer, 2004, vol. 301, pp. 251-264.

[33] A. Filippov, "Differential equations with discontinuous right-hand side," Amer. Math. Soc. Transl., vol. 42, no. 2, pp. 199-231, 1964.

[34] D. Shevitz and B. Paden, "Lyapunov stability theory of nonsmooth systems," IEEE Trans. Autom. Control, vol. 39, no. 9, pp. 1910-1914, Sep. 1994.

[35] F. H. Clarke, Optimization and Nonsmooth Analysis. Philadelphia, PA: SIAM, 1990.

[36] C. Mei, J. Cherng, and Y. Wang, "Active control of regenerative chatter during metal cutting process," J. Manufact. Sci. Eng., vol. 128, pp. 346-346, 2006

[37] Y. Altintas and M. Weck, "Chatter stability of metal cutting and grinding," CIRP Annals-Manufact. Technol., vol. 53, no. 2, pp. 619-642, 2004

[38] J. Wang and C. Su, "Two degree-of-freedom modeling and robust chatter control in metal cutting by piezoelectricity," in Proc. Amer. Control Conf., 2003, vol. 4, pp. 3044-3048. 